\title{
Seminar on Surgical Diseases of the Lymphatics in Childhood
}

\author{
Chairman: A. W. WILKINSON (London) \\ Moderators: M. H. Gough (London), A. H. Bill (Seattle), A. B. Wallace (Edinburgh), S. GodarT \\ (Brussels)
}

M. H. Gough illustrated the technique of lymphangiography with a film. General anaesthesia was necessary in children, and the lymphatics in the limbs were outlined by injecting $0.1 \mathrm{ml} .10 \%$ Patent Blue into a web space. Normal lymphatics were filled within a few minutes, but abnormal lymphatics in children may be difficult to identify, and an operating microscope may be of great assistance. In the foot or hand it may well be necessary to make an incision across the whole dorsum to identify the lymphatics. The lymphatics were then outlined by using a lipiodol in oily solution, the dose being restricted to $0.2 \mathrm{ml} . / \mathrm{kg}$. as a maximum, because deaths from pulmonary emboli have been reported with higher dosages. Screening with an image intensifier was carried out during the injection.

In children primary lymphoedema of the lower limbs is described according to the findings on lymphangiography. The lymphatics may be described as follows.

(1) Aplastic when they are absent or very minute.

(2) Hypoplastic when they may show as a solitary vessel passing all the way up the limb, or as a greatly distended plexus in the lower limb leading to regional gland groups which are deficient or absent.

(3) Hyperplastic when showing pooling of the dye as a result of inefficient valves.

Bilateral lymphangiograms are carried out routinely, as $50 \%$ of patients with unilateral lymphoedema have some abnormality on the other side.

M. H. Gough showed that $35 \%$ of patients with Turner's syndrome have lymphoedema. There may be superficial-to-deep connexions which limit the appearance and degree of lymphoedema, and oestrogen therapy seems to be related to the appearance of lymphoedema.

He then discussed the problem of chylous reflux, which had a good prognosis if it was associated with hypoplasia of the lymphatics, but a bad prognosis if it was associated with the large lymphatics and haemangiomata of the limbs. He stressed the problem of protein-losing enteropathy associated with mesenteric obstruction of the lymphatics. Chyle can leak more easily from the mucous membrane surface than from the skin.

He warned about the danger of removing inguinal swellings that might be part of the drainage system.
In the discussion which followed it was observed that normal lymphatics had a remarkable ability for regeneration, particularly after excision of radial scars in the limbs. Within the past few years lymphatic-venous communications had been observed apart from the wellknown connexions in the thorax.

A. W. Wilkinson described his experiences with 7 patients with protein-losing enteropathy. They had presented with abdominal distension and steatorrhoea-like stools, with swelling of both lower limbs. They had recognizable radiological appearances in the intestinal mucosa. At operation, chronic volvulus of the affected intestine was noticeable. 2 patients improved with local resection of the affected gut, 2 were treated with steroids with apparent clinical improvement, and 3 were not treated other than by raising the limbs, which cleared up the abnormal character of the stool.

A. H. Bill discussed the aetiology of hygroma following a study of 61 children. Of these, 21 were lymphangiomata only with multiple small cystic spaces, 23 had large cysts and were classified as hygromata, and the remaining 17 were of a mixed type. Lymphatic obstruction appeared to be present, the different forms depending on the type of tissue involved. When these cysts occurred in loose connective tissue and fat they tended to be large and relatively easily removed. When they occurred in muscle they tended to be small, multicystic, and extremely difficult to eradicate, though they could be reduced by multiple excisions, e.g. in the tongue. Treatment is by excision, if possible completely. Radiotherapy or the injection of sclerosing solutions are not advised. Overgrowth of the involved tissue may occur, e.g. the mandible or tongue, but the aetiology is unknown and infection may cause temporary enlargement.

A. B. Wallace discussed the nature of lymphatic oedema, emphasizing that extra vascular fluid leads to fibroplasia, and the increased risk of cellulitis.

He indicated that Milroy's disease did not always show progressive lymphoedema, illustrated by a patient who was cured following the local excision of involved tissue on the dorsum of the feet. He wondered whether lymphangiography could cause obstruction to lymph channels that were already patent. In primary lymphoedema he pointed out that Kinmonth had demonstrated 8 out of 15 children with abnormal capillary flow rates. Might an abnormal capillary filtration mechanism be a causative factor? He reminded the meeting of the 
condition of congenital lipoedema with associated excess of fat, oedema, and fibrous tissue in the subcutaneous tissues. Acquired lymphoedema could be allergic, inflammatory, or traumatic, and he illustrated examples of these. Having found excision and grafting procedures, as outlined by Gibson and Tough, somewhat mutilating procedures, he now adopted Thomson's operation of inserting a skin flap alongside the main vessels in the thigh in trying to develop superficial-todeep lymphatic connexions.

S. Godart discussed the embryological significance of lymphangioma (published in full in this issue). Sabin's and Goetsch's original observations of centrifugal development of the peripheral lymphatics from the walls of the primary lymphatic sacs had been proved wrong.

Lymphatics develop as mesenchymal slits which appear in the 9 to $12 \mathrm{~mm}$. embryo in the reticulum of a rich venous plexus. They coalesce and form large lymphatic cavities which open secondarily into the venous system. These large cavities later decrease in size, become straight and narrow, and follow the direction of the veins. Lack of communications produces cystic hygromata, which are found in the same locations as foetal lymphatic sacs, e.g. cervical, mediastinal, or retroperitoneal regions. The multilocular smaller cavities are seen more peripherally than centrally.

P. R. Mellish (Vermont) described experiments in dogs, where the lymphatics draining a burnt area were cannulated. No bacteria appeared in the lymph collected from the animals with normal circulation, but a large flow of bacteria was obtained in those dogs whose arterial blood pressure had been reduced to $70 \mathrm{~mm}$. $\mathrm{Hg}$. 